\title{
OPEN Role of the IL33 and ILIRL1 pathway in the pathogenesis of Immunoglobulin A vasculitis
}

\author{
Diana Prieto-Peña ${ }^{1,21}$, Sara Remuzgo-Martínez ${ }^{1,21}$, Fernanda Genre ${ }^{1,21}$, \\ Verónica Pulito-Cueto ${ }^{1}$, Belén Atienza-Mateo ${ }^{1,2}$, Javier Llorca ${ }^{3}$, Belén Sevilla-Pérez ${ }^{4}$, \\ Norberto Ortego-Centeno ${ }^{5}$, Ana Marquez ${ }^{6,7}$, Leticia Lera-Gómez ${ }^{1}$, María Teresa Leonardo ${ }^{8}$, \\ Ana Peñalba ${ }^{8}$, Javier Narváez ${ }^{9}$, Luis Martín-Penagos ${ }^{10}$, Emilio Rodrigo ${ }^{10}$, \\ José A. Miranda-Filloy ${ }^{11}$, Luis Caminal-Montero ${ }^{12}$, Paz Collado ${ }^{13}$, Javier Sánchez Pérez ${ }^{14}$, \\ Diego de Argila ${ }^{14}$, Esteban Rubio ${ }^{15}$, Manuel León Luque ${ }^{15}$, Juan María Blanco-Madrigal ${ }^{16}$, \\ Eva Galíndez-Agirregoikoa ${ }^{16}$, Oreste Gualillo ${ }^{17}$, Javier Martín ${ }^{6}$, Santos Castañeda ${ }^{18}$, \\ Ricardo Blanco ${ }^{1}$, Miguel A. González-Gay ${ }^{1,19,20,22}$ \& Raquel López-Mejías ${ }^{1,22 \bowtie}$
}

Cytokines signalling pathway genes are crucial factors of the genetic network underlying the pathogenesis of Immunoglobulin-A vasculitis (IgAV), an inflammatory vascular condition. An influence of the interleukin (IL)33- IL1 receptor like (ILIRL)1 signalling pathway on the increased risk of several immune-mediated diseases has been described. Accordingly, we assessed whether the IL33-ILIRL1 pathway represents a novel genetic risk factor for IgAV. Three tag polymorphisms within IL33 (rs3939286, rs7025417 and rs7044343) and three within IL1RL1 (rs2310173, rs13015714 and rs2058660), that also were previously associated with several inflammatory diseases, were genotyped in 380 Caucasian IgAV patients and 845 matched healthy controls. No genotypes or alleles differences were observed between IgAV patients and controls when IL33 and ILIRLI variants were analysed independently. Likewise, no statistically significant differences were found in IL33 or IL1RL1 genotype and allele frequencies when IgAV patients were stratified according to the age at disease onset or to the presence/absence of gastrointestinal (GI) or renal manifestations. Similar results were disclosed when IL33 and ILIRLI haplotypes were compared between IgAV patients and controls and between

${ }^{1}$ Research Group On Genetic Epidemiology and Atherosclerosis in Systemic Diseases and in Metabolic Bone Diseases of the Musculoskeletal System, IDIVAL, Division of Rheumatology, Hospital Universitario Marqués de Valdecilla, Avenida Cardenal Herrera Oria s/n, 39011 Santander, Spain. 'López Albo' Post-Residency Programme, Hospital Universitario Marqués de Valdecilla, Santander, Spain. ${ }^{3}$ Epidemiology and Computational Biology Department, School of Medicine, Universidad de Cantabria, and CIBER Epidemiología y Salud Pública (CIBERESP), Santander, Spain. ${ }^{4}$ Division of Paediatrics, Hospital Universitario San Cecilio, Granada, Spain. ${ }^{5}$ Department of Medicine, Universidad de Granada, Granada, Spain. 'Instituto de Parasitología y Biomedicina 'López-Neyra', CSIC, PTS Granada, Granada, Spain. ${ }^{7}$ Systemic Autoimmune Disease Unit, Hospital Universitario Clínico San Cecilio, Instituto de Investigación Biosanitaria ibs.GRANADA, Granada, Spain. ${ }^{8}$ Division of Paediatrics, Hospital Universitario Marqués de Valdecilla, Santander, Spain. ${ }^{9}$ Division of Rheumatology, Hospital Universitario de Bellvitge, Barcelona, Spain. ${ }^{10}$ Division of Nephrology, Hospital Universitario Marqués de Valdecilla, IDIVAL-REDINREN, Santander, Spain. ${ }^{11}$ Division of Rheumatology, Hospital Universitario Lucus Augusti, Lugo, Spain. ${ }^{12}$ Division of Rheumatology, Hospital Universitario Central de Asturias, Instituto de Investigación Sanitaria del Principado de Asturias (ISPA), Oviedo, Spain. ${ }^{13}$ Division of Rheumatology, Hospital Universitario Severo Ochoa, Madrid, Spain. ${ }^{14}$ Department of Dermatology, Hospital Universitario de La Princesa, Madrid, Spain. ${ }^{15}$ Department of Rheumatology, Hospital Universitario Virgen del Rocío, Sevilla, Spain. ${ }^{16}$ Department of Rheumatology, Hospital Universitario de Basurto, Bilbao, Spain. ${ }^{17}$ SERGAS (Servizo Galego de Saude) and IDIS (Instituto de Investigación Sanitaria de Santiago), NEIRID Lab (Neuroendocrine Interactions in Rheumatology and Inflammatory Diseases), Research Laboratory 9, Hospital Clínico Universitario de Santiago, Santiago de Compostela, Spain. ${ }^{18}$ Department of Rheumatology, Hospital Universitario de La Princesa, IIS-Princesa, Madrid, Spain. ${ }^{19}$ School of Medicine, Universidad de Cantabria, Santander, Spain. ${ }^{20}$ Cardiovascular Pathophysiology and Genomics Research Unit, School of Physiology, Faculty of Health Sciences, University of the Witwatersrand, Johannesburg, South Africa. ${ }^{21}$ These authors contributed equally: Diana Prieto-Peña, Sara Remuzgo-Martínez and Fernanda Genre. ${ }^{22}$ These authors jointly supervised this work: Miguel A. González-Gay and Raquel López-Mejías. ${ }^{\circledR}$ email: rlopezmejias78@gmail.com 
IgAV patients stratified according to the clinical characteristics mentioned above. Our results suggest that the IL33-ILIRL1 signalling pathway does not contribute to the genetic network underlying IgAV.

Immunoglobulin-A vasculitis (IgAV), formerly called Henoch-Schönlein purpura (HSP), is an inflammatory small-sized blood vessel disease, more common in children and rarer but more serious in adults ${ }^{1-3}$. The defining pathophysiologic feature of this vasculitis is the IgA1-predominant immune deposits in the vessel walls ${ }^{1}$. Although IgAV usually involves the skin, joints and the gastrointestinal (GI) tract ${ }^{4-8}$, nephritis is also common in affected patients and constitutes the most feared complication of this vasculitis ${ }^{1-5}$. IgAV has a multifactorial aetiology in which genetics plays a relevant role in both the predisposition and clinical course of the disease $e^{6,9,10}$. In this regard, outside the human leukocyte antigen region, cytokines signalling pathway genes constitute a key component of the genetic network underlying IgAV $\mathrm{V}^{6,11-14}$.

Interleukin (IL)-33 is a cytokine that belongs to the IL- 1 family ${ }^{15}$. This molecule exerts its biological functions by binding to its receptor, IL-1 receptor like 1 (IL-1RL1) (also known as suppression of tumorigenicity 2 or ST2), a member of the Toll/IL-1 receptor superfamily ${ }^{16}$. This binding leads to the activation of mast cells and Th2 lymphocytes and, consequently, to the production of chemokines, pro-inflammatory and Th2-associated cytokines, as well as increased serum immunoglobulin levels ${ }^{15}$. In accordance with that, a pathogenic role of the IL-33 axis in autoimmunity has been described ${ }^{17-19}$. In addition, several lines of evidence demonstrate that genetic variants located both in the IL33 and IL1RL1 genes are implicated in the increased risk of numerous immune-mediated diseases ${ }^{20-28}$.

Taking into account all these considerations, this study aimed to determine, for the first time, the potential implication of the IL33-IL1RL1 signalling pathway in the pathogenesis of IgAV. For this purpose, we genotyped three tag genetic variants within IL33 and three tag polymorphisms within IL1RL1, which cover the major variability of these loci and that were previously associated with several inflammatory diseases, in the largest series of Caucasian IgAV patients ever assessed for genetic studies.

\section{Patients and methods}

Study population. A series of 380 unrelated Spanish patients of European ancestry who fulfilled Michel et al. criteria ${ }^{29}$ and/or the American College of Rheumatology classification criteria ${ }^{30}$ for IgAV-HSP and/or the 2012 revised International Chapel Hill Consensus Conference Nomenclature ${ }^{31}$ definition for IgAV were included in the present study. Centres involved in the recruitment of these patients included Hospital Universitario Marqués de Valdecilla (Santander), Hospital Universitario San Cecilio (Granada), Hospital Universitario de Bellvitge (Barcelona), Hospital Universitario Lucus Augusti (Lugo), Hospital Universitario Central de Asturias (Oviedo), Hospital Universitario Severo Ochoa and Hospital Universitario de La Princesa (Madrid), Hospital Universitario Virgen del Rocío (Sevilla) and Hospital Universitario de Basurto (Bilbao). Information on the main clinical features of these patients is displayed in Table 1. For GI manifestations, bowel angina was considered present if there was diffuse abdominal pain that worsened after meals or bowel ischemia usually with bloody diarrhoea. GI bleeding was defined as the presence of melena, haematochezia, or a positive test for occult blood in the stool. Renal manifestations were defined to be present if at least one of the following findings was observed: haematuria, proteinuria, or nephrotic syndrome at any time over the clinical course of the disease and/or renal sequelae (persistent renal involvement) at last follow-up.

In addition, a set of 845 sex and ethnically matched healthy controls without history of cutaneous vasculitis or any other autoimmune disease, constituted by blood donors from Hospital Universitario Marqués de Valdecilla (Santander) and National DNA Bank Repository (Salamanca), was also included in this study.

For experiments involving humans and the use of human blood samples, all the methods were carried out in accordance with the approved guidelines and regulations, according to the Declaration of Helsinki. All experimental protocols were approved by the Ethics Committees of clinical research of Cantabria (for Hospital Universitario Marqués de Valdecilla, Santander), Ethics Committee of clinical research of Granada (for Hospital Universitario San Cecilio, Granada), Ethics Committee of clinical research of Barcelona (for Hospital Universitario de Bellvitge, Barcelona), Ethics Committee of clinical research of Galicia (for Hospital Universitario Lucus Augusti, Lugo), Ethics Committee of clinical research of Asturias (for Hospital Universitario Central de Asturias, Oviedo), Ethics Committee of clinical research of Madrid (for Hospital Universitario Severo Ochoa and Hospital Universitario de La Princesa, Madrid), Ethics Committee of clinical research of Sevilla (for Hospital Universitario Virgen del Rocío, Sevilla) and Ethics Committee of clinical research of Euskadi (for Hospital Universitario de Basurto, Bilbao). Patients with IgAV and healthy controls signed an informed written consent before being included in the study.

Single nucleotide polymorphisms selection and genotyping methods. Three tag genetic variants within IL33 (rs3939286, rs7025417 and rs7044343) and three tag polymorphisms within IL1RL1 (rs2310173, rs13015714 and rs2058660) were selected in this study. Regarding IL33, rs3939286 is situated in an intergenic region close to IL33, whereas rs7025417 and rs7044343 are located in the promoter and in intron 5 of the gene, respectively. With respect to $I L 1 R L 1$, both rs2310173 and r2058660 are placed close to IL1RL1, while rs 13015714 is downstream of the gene. Interestingly, these six polymorphisms map and tag both IL33 and IL1RL1 regions as previously described ${ }^{20}$. In addition, the IL33 and IL1RL1 genetic variants selected in our study were previously associated with several immune-mediated diseases ${ }^{20-28}$. In particular, IL33 rs3939286 was previously implicated in the development of subclinical atherosclerosis in patients with rheumatoid arthritis (RA) ${ }^{20}$ as well as in the pathogenesis of inflammatory bowel (IBD) disease ${ }^{21}$ and asthma ${ }^{22}$, whereas IL33 rs7025417 was previously associated with coronary artery disease ${ }^{23}$, and rs7044343 with $\mathrm{RA}^{24}$. Regarding IL1RL1, a relevant role of rs 2310173 


\begin{tabular}{|c|c|}
\hline & $\%(\mathbf{n})$ \\
\hline Children (age $\leq 20$ years)/ adults (age $>20$ years) $(\mathrm{n})$ & $294 / 86$ \\
\hline Percentage of females & 47.9 \\
\hline Age at disease onset (years, median [IQR]) & $7(5-19)$ \\
\hline Duration of follow-up (years, median [IQR]) & $1(1-3)$ \\
\hline Palpable purpura and/or maculopapular rash & $100(380)$ \\
\hline Arthralgia and/or arthritis & $52.9(201)$ \\
\hline GI manifestations (if "a" and/or "b") & $53.2(202)$ \\
\hline (a) Bowel angina & $50.5(192)$ \\
\hline (b) GI bleeding & $16.3(62)$ \\
\hline Renal manifestations (if any of the following characteristics) & $36.1(137)$ \\
\hline (a) Haematuria* & $34.5(131)$ \\
\hline (b) Proteinuria* & $32.9(125)$ \\
\hline (c) Nephrotic syndrome $e^{*}$ & $5.5(21)$ \\
\hline (d) Renal sequelae (persistent renal involvement) ${ }^{* *}$ & $6.6(25)$ \\
\hline
\end{tabular}

Table 1. Main clinical features of the 380 patients with IgAV included in the study. IgAV: IgA vasculitis; IQR: interquartile range; GI: gastrointestinal. ${ }^{\star}$ At any time over the clinical course of the disease. ${ }^{\star *}$ At last follow-up.

in ankylosing spondylitis ${ }^{25}$ and ulcerative colitis ${ }^{26}$, as well as an association of rs13015714 with celiac disease ${ }^{27}$ and $\mathrm{IBD}^{21}$, and an influence of rs 2058660 on the development of Crohn's disease ${ }^{28}$ were previously reported.

Genomic deoxyribonucleic acid (DNA) from all the individuals included in the study was extracted from peripheral blood using standard procedures.

Patients with IgAV and healthy controls were genotyped for the six polymorphisms mentioned above using predesigned TaqMan 5' single-nucleotide polymorphism genotyping assays (C___2762168_10 for rs3939286, C_31940410_20 for rs7025417, C__29340326_10 for rs7044343, C__ 2676437_10 for rs2310173, C_31439507_10 for rs13015714 and C_11487892_10 for rs2058660).

Genotyping was performed in a QuantStudioTM 7 Flex Real-Time polymerase chain reaction system, according to the conditions recommended by the manufacturer (Applied Biosystems, Foster City, CA, USA).

Negative controls and duplicate samples were included to check the accuracy of the genotyping.

Statistical analyses. Genotype data were checked for deviation from Hardy-Weinberg equilibrium (HWE).

Differences in IL33 and IL1RL1 frequencies were evaluated between patients with IgAV and healthy controls and between patients with IgAV stratified according to specific clinical characteristics of the disease (age at disease onset or presence/absence of GI or renal manifestations).

First, each $I L 33$ and IL1RL1 polymorphism was analysed independently. Both genotype and allele frequencies were calculated and compared between the groups mentioned above by chi-square test. Strength of association was estimated using odds ratios (OR) and 95\% confidence intervals (CI).

Then, allelic combinations (haplotypes) of both IL33 and IL1RL1 polymorphisms were carried out. Haplotype frequencies were calculated by the Haploview v4.2 software (http://broad.mit.edu/mpg/haploview) and then compared between the groups mentioned above by chi-square test. Strength of association was estimated by OR and $95 \% \mathrm{CI}$.

$P$-values were two-tailed and those lower than 0.05 were considered as statistically significant. All analyses were performed with the STATA statistical software 12/SE (Stata Corp., College Station, TX, USA).

\section{Results}

Genotyping quality control. IL33 rs3939286, IL33 rs7025417, IL33 rs7044343, IL1RL1 rs2310173, IL1RL1 rs13015714 and IL1RL1 rs2058660 were in HWE.

The genotyping success rate for each polymorphism evaluated in this study was $99.9 \%$ for IL33 rs3939286, 99.4\% for IL33 rs7025417, 99.8\% for IL33 rs7044343, 100\% for IL1RL1 rs2310173, 99.6\% for IL1RL1 rs 13015714 and $99.8 \%$ for $I L 1 R L 1 \mathrm{rs} 2058660$.

Genotype and allele frequencies of each IL33 and IL1RL1 polymorphism were similar to that reported for populations of European origin in the 1000 Genomes Project (http://www.internationalgenome.org/).

Differences in IL33 and ILIRLI genotype and allele frequencies between patients with IgAV and healthy controls. In a first step, we compared genotype and allele frequencies of each IL33 and ILIRLI variant assessed independently between patients with IgAV and healthy controls.

As shown in Table 2, similar genotype and allele IL33 and IL1RL1 frequencies were observed in patients with IgAV when compared to healthy controls (Table 2).

Differences in IL33 and ILIRLI genotype and allele frequencies between patients with IgAV stratified according to specific clinical characteristics of the disease. Then, potential differences 


\begin{tabular}{|c|c|c|c|c|c|c|c|c|}
\hline \multicolumn{4}{|l|}{ Change } & \multicolumn{3}{|c|}{ Genotypes, \% (n) } & \multicolumn{2}{|c|}{ Alleles, \% (n) } \\
\hline Locus & SNP & $1 / 2$ & Samples set & $1 / 1$ & $1 / 2$ & $2 / 2$ & 1 & 2 \\
\hline \multirow[t]{2}{*}{ IL33 } & rs3939286 & $\mathrm{C} / \mathrm{T}$ & IgAV patients & $49.1(186)$ & 40.9 (155) & $10.0(38)$ & $69.5(527)$ & $30.5(231)$ \\
\hline & & & Healthy controls & 49.0 (414) & $41.4(350)$ & $9.6(81)$ & 69.7 (1178) & $30.3(512)$ \\
\hline \multirow[t]{2}{*}{ IL33 } & rs7025417 & $\mathrm{T} / \mathrm{C}$ & IgAV patients & $68.1(254)$ & $29.5(110)$ & $2.4(9)$ & $82.8(618)$ & $17.2(128)$ \\
\hline & & & Healthy controls & $70.8(598)$ & 25.9 (219) & $3.3(28)$ & 83.7 (1415) & $16.3(275)$ \\
\hline \multirow[t]{2}{*}{ IL33 } & rs7044343 & $\mathrm{T} / \mathrm{C}$ & IgAV patients & $42.3(160)$ & 42.1 (159) & $15.6(59)$ & $63.4(479)$ & \begin{tabular}{|l|}
$36.6(277)$ \\
\end{tabular} \\
\hline & & & Healthy controls & $44.5(376)$ & 43.9 (371) & $11.6(98)$ & $66.4(1123)$ & $33.6(567)$ \\
\hline \multirow[t]{2}{*}{ ILIRL1 } & rs2310173 & G/T & IgAV patients & $29.2(111)$ & $46.1(175)$ & $24.7(94)$ & $52.2(397)$ & $47.8(363)$ \\
\hline & & & Healthy controls & $30.2(255)$ & 46.7 (395) & $23.1(195)$ & $53.6(905)$ & $46.4(785)$ \\
\hline \multirow[t]{2}{*}{ ILIRL1 } & rs13015714 & $\mathrm{T} / \mathrm{G}$ & IgAV patients & $56.3(211)$ & 39.5 (148) & $4.3(16)$ & $76.0(570)$ & $24.0(180)$ \\
\hline & & & Healthy controls & $57.2(483)$ & $37.2(314)$ & $5.7(48)$ & $75.7(1280)$ & $24.3(410)$ \\
\hline \multirow[t]{2}{*}{ IL1RL1 } & rs2058660 & $\mathrm{A} / \mathrm{G}$ & IgAV patients & $56.9(215)$ & 38.6 (146) & $4.5(17)$ & $76.2(576)$ & $23.8(180)$ \\
\hline & & & Healthy controls & 56.7 (479) & $37.5(317)$ & $5.8(49)$ & $75.4(1275)$ & 24.6 (415) \\
\hline
\end{tabular}

Table 2. Genotype and allele frequencies of IL33 and IL1RL1 genes in patients with IgAV and healthy controls. IgAV: IgA vasculitis; SNP: single nucleotide polymorphism. No statistically significant differences in the IL33 and IL1RL1 genotype and allele frequencies were disclosed when patients with IgAV were compared to healthy controls ( $p \geq 0.05$ in all the cases).

in the genotype and allele frequencies of each IL33 and IL1RL1 genetic variant were evaluated between patients with IgAV stratified according to specific clinical characteristics of the disease.

Given that IgAV is often a benign and self-limited pathology in children and a more severe condition in adults, we assessed if potential differences in IL33 and IL1RL1 could exist in IgAV patients stratified according to the age at disease onset. With this respect, and as shown in Table 3, similar genotype and allele IL33 and IL1RL1 frequencies were disclosed when children (age $\leq 20$ years) were compared to adults (age $>20$ years) (Table 3 ).

In addition, we compared genotype and allele frequencies of IL33 and ILIRL1 in patients with IgAV stratified according to the presence/absence of GI or renal manifestations. Accordingly, similar genotype and allele IL33 and IL1RL1 frequencies were disclosed when patients with IgAV who developed GI manifestations were compared to those who did not exhibit these complications (Table 3). This was also the case when patients with IgAV were stratified according to the presence/absence of renal manifestations (Table 3).

Haplotype analyses of IL33 and ILIRL1. In a further step, we also examined whether haplotype frequencies of both IL33 and IL1RL1 differed between patients with IgAV and healthy controls as well as between patients with IgAV stratified according to the specific clinical characteristics of the disease mentioned above.

The haplotype analysis did not yield additional information since IL33 and ILIRL1 haplotypes frequencies were similar in patients with IgAV when compared to healthy controls (Table 4). This was also the case when patients with IgAV were stratified according to the age at disease onset and the presence/absence of GI or renal manifestations (Supplementary Tables S1-S3 online).

\section{Discussion}

Several pieces of evidence clearly indicate that a common genetic background may underlie different inflammatory diseases ${ }^{32,33}$. In this regard, the IL33-IL1RL1 signalling pathway is described as a shared genetic risk factor for numerous immune-related diseases ${ }^{20-28}$.

Based on these considerations, in this study we aimed to determine whether the IL33-IL1RL1 signalling pathway is also implicated in the pathogenesis of IgAV, a small-vessel vasculitis in which cytokines signalling pathway genes are considered as an essential component in its etiology $\mathrm{y}^{6,11-14}$. For that purpose, six polymorphisms within the IL33-IL1RL1 signalling pathway, which cover most of the variability of both IL33 and IL1RL1 regions and that were previously related to numerous immune-mediated diasease ${ }^{20-28}$, were evaluated in the largest series of Caucasian patients with IgAV ever assessed for genetic studies. Our results showed no influence of the IL33-IL1RL1 signalling pathway on the susceptibility to IgAV when we studied each of the polymorphisms separately. Moreover, when we analysed $I L 33$ genetic variants as well as $I L 1 R L 1$ polymorphisms together conforming haplotypes, our results revealed a lack of association between the IL33-IL1RL1 signalling pathway and IgAV susceptibility. Furthermore, no specific association of IL33 and IL1RL1 polymorphisms (assessed independently or combined conforming haplotypes) with clinical features of IgAV was observed in our study, indicating that this pathway does not represent a risk factor for the severity of the disease.

Data on the potential effect of the IL33-IL1RL1 signalling pathway on vasculitis were scarce so far. A study performed in Caucasians evaluated the role of this pathway in the susceptibility to giant cell arteritis (GCA) ${ }^{34}$, another primary systemic vasculitis that involves large and middle-sized blood vessels ${ }^{31,35}$. In keeping with our results, no influence of IL33 rs3939286, IL33 rs7044343, IL1RL1 rs2310173, IL1RL1 rs13015714 and IL1RL1 rs 2058660 on GCA was detected ${ }^{34}$. By contrast, a consistent association between IL33 rs7025417 and GCA was 


\begin{tabular}{|c|c|c|c|c|c|c|}
\hline \multirow[b]{2}{*}{ Polymorphism } & \multicolumn{2}{|c|}{ Children (Age $\leq 20$ years) } & \multicolumn{2}{|c|}{ GI manifestations } & \multicolumn{2}{|c|}{ Renal manifestations } \\
\hline & Yes $(n=294)$ & No $(n=86)$ & Yes $(n=202)$ & No $(n=178)$ & Yes $(n=137)$ & No $(n=243)$ \\
\hline \multicolumn{7}{|l|}{ IL33 rs3939286 } \\
\hline $\mathrm{CC}$ & $47.8(140)$ & $53.5(46)$ & $47.3(95)$ & $51.1(91)$ & $48.5(66)$ & $49.4(120)$ \\
\hline CT & $42.0(123)$ & $37.2(32)$ & $42.3(85)$ & $39.3(70)$ & $39.7(54)$ & $41.6(101)$ \\
\hline TT & $10.2(30)$ & $9.3(8)$ & $10.4(21)$ & $9.6(17)$ & $11.8(16)$ & $9.1(22)$ \\
\hline $\mathrm{C}$ & $68.8(403)$ & $72.1(124)$ & $68.4(275)$ & $70.8(252)$ & $68.4(186)$ & $70.2(341)$ \\
\hline $\mathrm{T}$ & $31.2(183)$ & $27.9(48)$ & $31.6(127)$ & $29.2(104)$ & $31.6(86)$ & $29.8(145)$ \\
\hline \multicolumn{7}{|l|}{ IL33 rs7025417 } \\
\hline $\mathrm{TT}$ & $66.6(191)$ & $73.3(63)$ & $68.2(135)$ & $68.0(119)$ & $69.9(93)$ & $67.1(161)$ \\
\hline TC & $31.0(89)$ & $24.4(21)$ & $28.8(57)$ & $30.3(53)$ & $27.1(36)$ & $30.8(74)$ \\
\hline $\mathrm{CC}$ & $2.4(7)$ & $2.3(2)$ & $3.0(6)$ & $1.7(3)$ & $3.0(4)$ & $2.1(5)$ \\
\hline $\mathrm{T}$ & $82.1(471)$ & $85.5(147)$ & $82.6(327)$ & $83.1(291)$ & $83.5(222)$ & $82.5(396)$ \\
\hline C & $17.9(103)$ & $14.5(25)$ & $17.4(69)$ & $16.9(59)$ & $16.5(44)$ & $17.5(84)$ \\
\hline \multicolumn{7}{|l|}{ IL33 rs7044343 } \\
\hline TT & $44.2(129)$ & $36.0(31)$ & $43.9(88)$ & $40.7(72)$ & $44.5(61)$ & $41.1(99)$ \\
\hline TC & $39.4(115)$ & $51.2(44)$ & $39.8(80)$ & $44.6(79)$ & $39.4(54)$ & $43.6(105)$ \\
\hline $\mathrm{CC}$ & $16.4(48)$ & $12.8(11)$ & $16.4(33)$ & $14.7(26)$ & $16.1(22)$ & $15.4(37)$ \\
\hline $\mathrm{T}$ & $63.9(373)$ & $61.6(106)$ & $63.7(256)$ & $63.0(223)$ & $64.2(176)$ & $62.9(303)$ \\
\hline $\mathrm{C}$ & $36.1(211)$ & $38.4(66)$ & $36.3(146)$ & $37.0(131)$ & $35.8(98)$ & $37.1(179)$ \\
\hline \multicolumn{7}{|c|}{ IL1RL1 rs2310173 } \\
\hline GG & $29.6(87)$ & $27.9(24)$ & $30.2(61)$ & $28.1(50)$ & $32.1(44)$ & $27.6(67)$ \\
\hline GT & $45.2(133)$ & $48.8(42)$ & $45.0(91)$ & $47.2(84)$ & $43.1(59)$ & $47.7(116)$ \\
\hline TT & $25.2(74)$ & $23.3(20)$ & $24.8(50)$ & $24.7(44)$ & $24.8(34)$ & $24.7(60)$ \\
\hline G & $52.2(307)$ & $52.3(90)$ & $52.7(213)$ & $51.7(184)$ & $53.6(147)$ & $51.4(250)$ \\
\hline $\mathrm{T}$ & $47.8(281)$ & $47.7(82)$ & $47.3(191)$ & $48.3(172)$ & $46.4(127)$ & $48.6(236)$ \\
\hline \multicolumn{7}{|c|}{ IL1RL1 rs13015714 } \\
\hline $\mathrm{TT}$ & $55.5(161)$ & $58.8(50)$ & $57.3(114)$ & $55.1(97)$ & $61.8(84)$ & $53.1(127)$ \\
\hline TG & $40.0(116)$ & $37.6(32)$ & $40.2(80)$ & $38.6(68)$ & $33.8(46)$ & $42.7(102)$ \\
\hline GG & $4.5(13)$ & $3.5(3)$ & $2.5(5)$ & $6.3(11)$ & $4.4(6)$ & $4.2(10)$ \\
\hline $\mathrm{T}$ & $75.5(438)$ & $77.6(132)$ & $77.4(308)$ & $74.4(262)$ & $78.7(214)$ & $74.5(356)$ \\
\hline G & $24.5(142)$ & $22.4(38)$ & $22.6(90)$ & $25.6(90)$ & $21.3(58)$ & $25.5(122)$ \\
\hline \multicolumn{7}{|c|}{ IL1RL1 rs2058660 } \\
\hline AA & $56.3(165)$ & $58.8(50)$ & $57.9(117)$ & $55.7(98)$ & $62.2(84)$ & $53.9(131)$ \\
\hline AG & $39.2(115)$ & $36.5(31)$ & $39.1(79)$ & $38.1(67)$ & $31.9(43)$ & $42.4(103)$ \\
\hline GG & $4.4(13)$ & $4.7(4)$ & $3.0(6)$ & $6.3(11)$ & $5.9(8)$ & $3.7(9)$ \\
\hline A & $75.9(445)$ & $77.1(131)$ & $77.5(313)$ & $74.7(263)$ & $78.1(211)$ & $75.1(365)$ \\
\hline G & $24.1(141)$ & $22.9(39)$ & $22.5(91)$ & $25.3(89)$ & $21.9(59)$ & $24.9(121)$ \\
\hline
\end{tabular}

Table 3. Genotype and allele frequencies of IL33 and IL1RL1 genes in patients with IgAV stratified according to clinical characteristics of the disease. IgAV: IgA vasculitis; GI: gastrointestinal. No statistically significant differences in the $I L 33$ and $I L 1 R L 1$ genotype and allele frequencies were disclosed between patients with IgAV stratified according to the age at disease onset, the presence/absence of GI or renal manifestations $(p \geq 0.05$ in all the cases).

found, pointing out this polymorphism as a novel genetic risk factor for GCA susceptibility ${ }^{34}$. In addition, the potential role of an IL33 polymorphism (rs1342326) in the pathogenesis of Behçet's disease, a condition affecting blood vessels of all sizes and types ${ }^{31}$, was assessed in Iranians ${ }^{36}$. With this respect, an effect of this genetic variant in the susceptibility to this disease was disclosed ${ }^{36}$.

The results derived from our study are of potential clinical relevance. Vasculitides comprises a heterogeneous group of autoimmune diseases that often have overlapping clinical and pathological manifestations ${ }^{37}$. Although their complex aetiology is far from being completely understood, differences among them in molecular terms have been postulated ${ }^{38}$. Our data support this hypothesis since no implication of the IL33-IL1RL1 signalling pathway in the pathogenesis of IgAV in Caucasians was disclosed, unlike GCA ${ }^{34}$. 


\begin{tabular}{|l|l|l|l|l|}
\hline \multicolumn{2}{|l|}{ IL33 haplotypes } & \multirow{2}{*}{} & \multicolumn{1}{l}{} \\
\cline { 1 - 3 } rs3939286 & rs7025417 & rs7044343 & $\boldsymbol{p}$ & OR (95\% CI) \\
\hline C & T & T & - & Ref \\
\hline C & T & C & 0.26 & $1.15(0.89-1.47)$ \\
\hline T & T & T & 0.77 & $1.04(0.78-1.40)$ \\
\hline T & T & C & 0.35 & $1.15(0.85-1.58)$ \\
\hline T & C & T & 0.84 & $1.04(0.68-1.58)$ \\
\hline C & C & T & 0.53 & $1.14(0.74-1.71)$ \\
\hline IL1RL1 haplotypes & & & \\
\hline rs2310173 & rs13015714 & rs2058660 & $\boldsymbol{p}$ & OR (95\% CI) \\
\hline G & T & A & - & Ref \\
\hline T & T & A & 0.10 & $1.19(0.96-1.46)$ \\
\hline T & G & G & 0.50 & $0.91(0.70-1.19)$ \\
\hline G & G & G & 0.16 & $1.26(0.90-1.77)$ \\
\hline
\end{tabular}

Table 4. Haplotype analysis of $I L 33$ and $I L 1 R L 1$ genes between patients with IgAV and healthy controls. IgAV: IgA vasculitis; OR: Odds Ratio; CI: confidence interval. Haplotypes of IL33 and ILIRL1 with a frequency higher than $5 \%$ are shown.

In summary, based on a large series of Caucasian patients, our results suggest that the IL33-IL1RL1 signalling pathway does not contribute to the genetic network underlying IgAV.

Received: 25 May 2021; Accepted: 30 July 2021

Published online: 09 August 2021

\section{References}

1. González-Gay, M. A. \& García-Porrúa, C. Epidemiology of the vasculitides. Rheum. Dis. Clin. North Am. 27, 729-749 (2001).

2. Calviño, M. C. et al. Henoch-Schönlein purpura in children from northwestern Spain: A 20-year epidemiologic and clinical study. Medicine (Baltimore) 80, 279-290 (2001).

3. García-Porrúa, C. et al. Henoch-Schönlein purpura in children and adults: Clinical differences in a defined population. Semin Arthritis Rheum. 32, 149-156 (2002).

4. Calvo-Río, V. et al. Henoch-Schönlein purpura in northern Spain: Clinical spectrum of the disease in 417 patients from a single center. Medicine (Baltimore) 93, 106-113 (2014).

5. González-Gay, M. A., Blanco, R. \& Castañeda, S. Henoch-Schönlein purpura (IgA vasculitis): The paradox of the different incidence and clinical spectrum in children and adults. Clin. Exp. Rheumatol. 35(Suppl 103), 3-4 (2017).

6. López-Mejías, R. et al. Genetics of immunoglobulin-A vasculitis (Henoch-Schönlein purpura): An updated review. Autoimmun. Rev. 17, 301-315 (2018).

7. González-Gay, M. A. \& García-Porrúa, C. Systemic vasculitis in adults in Northwestern Spain, 1988-1997: Clinical and epidemiologic aspects. Med. (Baltimore) 78, 292-308 (1999).

8. Calvo-Río, V. et al. Relapses in patients with Henoch-Schönlein purpura: Analysis of 417 patients from a single center. Medicine (Baltimore) 95, 4217 (2016).

9. Wyatt, R. J. \& Julian, B. A. IgA nephropathy. N Engl. J. Med. 368, 2402-2414 (2013).

10. López-Mejías, R. et al. A genome-wide association study suggests the HLA Class II region as the major susceptibility locus for IgA vasculitis. Sci. Rep. 11, 5088 (2017).

11. López-Mejías, R. et al. Interleukin 1 beta (IL1ß) rs16944 genetic variant as a genetic marker of severe renal manifestations and renal sequelae in Henoch-Schönlein purpura. Clin. Exp. Rheumatol. 34, S84-S88 (2016).

12. Amoli, M. M. et al. Interleukin 1 receptor antagonist gene polymorphism is associated with severe renal involvement and renal sequelae in Henoch-Schönlein purpura. J. Rheumatol. 29, 1404-1407 (2002).

13. Amoli, M. M. et al. Interleukin 8 gene polymorphism is associated with increased risk of nephritis in cutaneous vasculitis. $J$. Rheumatol. 29, 2367-2370 (2002).

14. Amoli, M. M. et al. Interleukin 1beta gene polymorphism association with severe renal manifestations and renal sequelae in Henoch-Schönlein purpura. J. Rheumatol. 31, 295-298 (2004).

15. Schmitz, J. et al. IL-33, an interleukin-1-like cytokine that signals via the IL-1 receptor-related protein ST2 and induces T helper type 2-associated cytokines. Immunity 23, 479-490 (2005).

16. Chackerian, A. A. et al. IL-1 receptor accessory protein and ST2 comprise the IL-33 receptor complex. J. Immunol. 179, 2551-2555 (2007).

17. Theoharides, T. C. et al. IL-33 augments substance P-induced VEGF secretion from human mast cells and is increased in psoriatic skin. Proc. Natl. Acad. Sci. USA. 107, 4448-4453 (2010).

18. Xu, D. et al. IL-33 exacerbates antigeninduced arthritis by activating mast cells. Proc. Natl. Acad. Sci. USA. 105, 10913-10918 (2008).

19. Demyanets, S. et al. Interleukin-33 induces expression of adhesion molecules and inflammatory activation in human endothelial cells and in human atherosclerotic plaques. Arterioscler Thromb. Vasc. Biol. 31, 2080-2089 (2011).

20. López-Mejías, R. et al. Protective role of the interleukin 33 rs3939286 gene polymorphism in the development of subclinical atherosclerosis in rheumatoid arthritis patients. PLoS ONE 10, 0143153 (2015).

21. Latiano, A. et al. Associations between genetic polymorphisms in IL-33, IL1R1 and risk for inflammatory bowel disease. PLoS ONE 8, e62144 (2013).

22. Gudbjartsson, D. F. et al. Sequence variants affecting eosinophil numbers associate with asthma and myocardial infarction. Nat Genet. 41, 342-347 (2009). 
23. Tu, X. et al. The IL-33-ST2L pathway is associated with coronary artery disease in a Chinese Han population. Am. J. Hum. Genet. 93, 652-660 (2013).

24. Li, C. et al. Genetic variant in IL33 is associated with susceptibility to rheumatoid arthritis. Arthritis Res. Ther. 16, R105 (2014).

25. Reveille, J. D. et al. Genome-wide association study of ankylosing spondylitis identifies non-MHC susceptibility loci. Nat. Genet. 42, 123-127 (2010).

26. Anderson, C. A. et al. Metaanalysis identifies 29 additional ulcerative colitis risk loci, increasing the number of confirmed associations to 47. Nat. Genet. 43, 246-252 (2011).

27. Hunt, K. A. et al. Newly identified genetic risk variants for celiac disease related to the immune response. Nat. Genet. 40, 395-402 (2008).

28. Franke, A. et al. Genome-wide meta-analysis increases to 71 the number of confirmed Crohn's disease susceptibility loci. Nat. Genet. 42, 1118-1125 (2010).

29. Michel, B. A. et al. Hypersensitivity vasculitis and Henoch-Schönlein purpura: a comparison between the 2 disorders. J. Rheumatol. 19, 721-728 (1992).

30. Mills, J. A. et al. The American College of Rheumatology 1990 criteria for the classification of Henoch-Schönlein purpura. Arthritis Rheum. 33, 1114-1121 (1990).

31. Jennette, J. C. et al. 2012 revised international Chapel Hill consensus conference nomenclature of vasculitides. Arthritis Rheum. 65, 1-11 (2013).

32. Wandstrat, A. \& Wakeland, E. The genetics of complex autoimmune diseases: non-MHC susceptibility genes. Nat Immunol. 2 , 802-809 (2001).

33. Zhernakova, A., Van Diemen, C. C. \& Wijmenga, C. Detecting shared pathogenesis from the shared genetics of immune-related diseases. Nat. Rev. Genet. 10, 43-55 (2009).

34. Márquez, A. et al. A candidate gene approach identifies an IL33 genetic variant as a novel genetic risk factor for GCA. PLoS ONE 9, e113476 (2014).

35. Gonzalez-Gay, M. A. et al. Epidemiology of giant cell arteritis and polymyalgia rheumatica. Arthritis Rheum. 61, 1454-1461 (2009).

36. Talei, M. et al. Interleukin-33 gene expression and rs1342326 polymorphism in Behçet's disease. Immunol. Lett. 212, 120-124 (2019).

37. Jennette, J. C. et al. Nomenclature of systemic vasculitides. Proposal of an international consensus conference. Arthritis Rheum. 37, 187-192 (1994).

38. Carmona, F. D. et al. Genetic basis of vasculitides with neurologic involvement. Neurol. Clin. 37, $219-234$ (2019).

\section{Acknowledgements}

We are indebted to the patients and healthy controls for their essential collaboration to this study. We also thank the National DNA Bank Repository (Salamanca) for supplying part of the control samples. This study was supported by European Union FEDER funds and 'Fondo de Investigaciones Sanitarias' (Grant PI18/00042) from 'Instituto de Salud Carlos III' (ISCIII, Health Ministry, Spain). DP-P is a recipient of a Río Hortega programme fellowship from the ISCIII, co-funded by the European Social Fund (ESF, 'Investing in your future') (Grant Number CM20/00006). SR-M is supported by funds of the RETICS Program (RD16/0012/0009) (ISCIII, cofunded by the European Regional Development Fund (ERDF)). VP-C is supported by a pre-doctoral grant from IDIVAL (PREVAL 18/01). BA-M is a recipient of a 'López Albo' Post-Residency Programme funded by Servicio Cántabro de Salud. LL-G is supported by funds from IDIVAL (INNVAL20/06). OG is staff personnel of Xunta de Galicia (Servizo Galego de Saude (SERGAS)) through a research-staff stabilization contract (ISCIII/SERGAS) and his work is funded by ISCIII and the European Union FEDER fund (Grant Numbers RD16/0012/0014 (RIER) and PI17/00409). He is beneficiary of project funds from the Research Executive Agency (REA) of the European Union in the framework of MSCA-RISE Action of the H2020 Programme, project 734899-Olive-Net. RL-M is a recipient of a Miguel Servet type I programme fellowship from the ISCIII, co-funded by ESF ('Investing in your future') (Grant Number CP16/00033).

\section{Author contributions}

D.P.-P., S.R.-M. and F.G. participated in the design of the study, data analysis and helped to draft the manuscript. V.P.-C., B.A.-M. and L.L.-G. have been involved in the acquisition, interpretation of data and coordination and helped to draft the manuscript. J.L. participated in the analysis and interpretation of the data and has been involved in revising the manuscript critically for important intellectual content. B.S.-P., N.O.-C., M.T.L., A.P., J.N., L.M.-P., E.R., J.A.M.-F., L.C.-M., P.C., J.S.P., D.A., E.R., M.L.L., J.M.B.-M., E.G.-A., S.C. and R.B. have been involved in the recruitment of patients, interpretation of data and coordination and helped to draft the manuscript. OG and JM have been involved in the interpretation of data and coordination and helped to draft the manuscript. M.A.G.-G. and R.L.-M. have made substantial contributions to the conception and design of the study, acquisition of data, coordination and helped to draft the manuscript and have given final approval of the version to be published. All authors have read and approved the manuscript for publication.

\section{Competing interests}

The authors declare no competing interests.

\section{Additional information}

Supplementary Information The online version contains supplementary material available at https://doi.org/ 10.1038/s41598-021-95762-5.

Correspondence and requests for materials should be addressed to R.L.-M.

Reprints and permissions information is available at www.nature.com/reprints.

Publisher's note Springer Nature remains neutral with regard to jurisdictional claims in published maps and institutional affiliations. 
(c) (i) Open Access This article is licensed under a Creative Commons Attribution 4.0 International cc) License, which permits use, sharing, adaptation, distribution and reproduction in any medium or format, as long as you give appropriate credit to the original author(s) and the source, provide a link to the Creative Commons licence, and indicate if changes were made. The images or other third party material in this article are included in the article's Creative Commons licence, unless indicated otherwise in a credit line to the material. If material is not included in the article's Creative Commons licence and your intended use is not permitted by statutory regulation or exceeds the permitted use, you will need to obtain permission directly from the copyright holder. To view a copy of this licence, visit http://creativecommons.org/licenses/by/4.0/.

(C) The Author(s) 2021 